\title{
Comparing treatment options for chronic inflammatory neuropathies and choosing the right treatment plan
}

Eduardo Nobile-Orazio, MD, PhD, ${ }^{1,2}$ Francesca Gallia, $M D,{ }^{2}$ Fabrizia Terenghi MD, ${ }^{2}$ Mariangela Bianco, $M D,^{2}$

${ }^{1}$ Department of Medical Biotechnology and Translational Medicine (BIOMETRA), University of Milan, ${ }^{2}$ Neuromuscular and Neuroimmunology Service, IRCCS Humanitas Clinical Institute, Rozzano, Milan, ITALY

Address Correspondence to:

Eduardo Nobile-Orazio, MD, PhD, FAAN, Department of Medical Biotechnology and Translational Medicine (BIOMETRA), Milan University, Neuromuscular and Neuroimmunology Service, IRCCS Humanitas Clinical Institute, Via Manzoni 56, 20089, Rozzano, Milan, ITALY

Tel. No: +390282242209; Fax. No: +390282242298; email: eduardo.nobile@unimi.it

Key words: Anti-MAG neuropathy; CIDP; immune therapies; IVIg; MMN; neuropathy; steroids; plasma exchange; Rituximab; SCIg; therapy 


\section{Conflicts of interest}

EN-O reports personal compensation for serving on the Steering Committee or Advisory Board of: Baxter, Italy; CSL Behring, Italy and USA; Kedrion Biopharma, Italy; LFB, France; Novartis, Switzerland; UCB, UK. He has received honoraria for lecturing from Baxter, USA and Italy, CSL Behring, Italy, Kedrion Biopharma, Italy, Grifols, Spain and travel supports for Scientific Meetings from CSL Behring, Italy and Kedrion Biopharma, Italy. All compensations and supports are outside the submitted work.

FG, FT and MB received travel supports for Scientific Meetings from CSL Behring, Italy and Kedrion Biopharma, Italy. 


\section{ABSTRACT}

Introduction: Chronic inflammatory neuropathies are disorders caused by an immune response to peripheral nerve. They include chronic inflammatory demyelinating polyradiculoneuropathy (CIDP), multifocal motor neuropathy (MMN) and neuropathy associated with anti-MAG IgM monoclonal gammopathy and other less frequent neuropathies. Several immune therapies have been proven to be effective in these neuropathies even if the best therapeutic option is still unsettled.

Areas covered: We reviewed the literature to compare the efficacy and safety of currently used immune therapies in these neuropathies. We also analysed the effect of other immune suppressive agents and of biological agents including rituximab, eculizumab, natalizumab, alemtuzumab and fingolimod that were found effective in other autoimmune diseases.

Expert commentary: Despite the reported efficacy of a number of new immune therapies in some patients with immune mediated neuropathies, their efficacy has not been so far confirmed in randomized controlled studies. High-dose intravenous immunoglobulin (IVIg) (and subcutaneous immunoglobulin [SCIg] for maintenance treatment), steroids and plasma exchange remain the only therapy of proven efficacy in CIDP, IVIg in MMN and, with certain limits, rituximab and, occasionally plasma exchange in neuropathy associated with anti-MAG antibodies. New biological agents are also on the horizon but their efficacy needs to be proved in controlled studies. 


\section{INTRODUCTION}

Chronic inflammatory neuropathies are an heterogeneous group of disorders deemed to be caused by an autoimmune reaction against peripheral nerve. They mainly include chronic inflammatory demyelinating polyradiculoneuropathy (CIDP) and its variants, multifocal motor neuropathy (MMN) and neuropathy associated with an IgM monoclonal gammopathy with antibody activity against the myelin-associated glycoprotein (MAG). Most patients with these neuropathies respond to immune therapies even if their effect varies in the different forms and among the different variants of CIDP. We review the effect of therapies in these neuropathies and suggest a therapeutic approach to these neuropathies.

\section{SEARCH STRATEGY}

We performed a search of available studies up to April 2014 on the Cochrane Neuromuscular Specialized Register and the Cochrane Central Register of Controlled Trials (CENTRAL) on the Cochrane Library and on Medline. We used the search terms steroids, corticosteroids, prednisone, prednisolone, methylprednisolone, dexamethasone, high dose intravenous immunoglobulin, IVIg, immunoglobulins, subcutaneous immunoglobulin, SCIg, azathioprine, methotrexate, interferon, cyclosporin, rituximab, cyclophosphamide, alentuzumab, mycophenolate, fingolimod, natalizumab, immune therapy, chronic inflammatory demyelinating polyradiculoneuropathy or polyneuropathy or polyradiculopathy, CIDP, polyneuropathy, neuropathy and polyneuritis. We provided our personal view on treatment strategies under the sections “How we treat ..” based on available guidelines and on our personal experience. 


\section{CHRONIC INFLAMMATORY DEMYELINATING \\ POLYRADICULONEUROPATHY}

\section{1.a Clinical features and Diagnosis}

Chronic inflammatory demyelinating polyradiculoneuropathy (CIDP) is a chronic and disabling disorder of the peripheral nervous system [1, 2] with a prevalence ranging from 0.8 to 8.9/100,000 [3-5]. CIDP may affect individuals at any age even if in most series the mean age of onset is around 40-50 years. There is a general consensus that CIDP is an immune mediated disorder affecting peripheral nerve myelin even if the target of this immune response is rarely identified [6, 7]. CIDP may have a chronic progressive or a relapsing course affecting the sensory and motor nerves while an autonomic impairment is uncommon. A number of atypical variants of CIDP have been described including Lewis-Sumner syndrome (multifocal acquired demyelinating sensory and motor - MADSAM- neuropathy), distal acquired demyelinating symmetric (DADS) neuropathy, purely motor, purely sensory and focal CIDP [2, 7]. CIDP is a severe disease with over $50 \%$ of the patients having a severe disability in the course of the disease leading to inability to walk without support and approximately $10 \%$ eventually become persistently disabled or die because of the illness [3]. Even if the diagnosis of CIDP is often easy in the clinical practice, the use of expensive therapies for this disease and the description of a few variants has led to the proposal of at least 15 diagnostic criteria [8] to define the boundaries of this neuropathy. This in order to avoid the inappropriate use of therapies in patients who might be not affected by CIDP without excluding patients who might benefit from immune treatment. The recently revised criteria of the EFNS/PNS [9] have the advantage of including patients with typical and atypical presentation of CIDP and to include patients with a demyelinating abnormalities in a single nerve when other supportive criteria for the diagnosis of CIDP are present. 
When CIDP is suspected based on a relapsing or progressive impairment that might not be as slow as usually observed in other chronic acquired neuropathy and that might also affect proximal strength, motor and sensory nerve conduction studies are considered to be sufficient for the diagnosis of CIDP [9]. When these studies do not show signs of multifocal demyelination with reduced conduction velocities, increased latencies and conduction block, the diagnosis of CIDP may be supported by cerebrospinal fluid examination showing increased proteins with normal cells in over $80 \%$ of the patients. Magnetic resonance imaging (MRI) and nerve ultrasound (US) may be also helpful by revealing gadolinium enhancement or hypertrophy of spinal roots, brachial or lumbosacral plexus. Sural nerve biopsy is often unnecessary in most patients with suspected CIDP, since the findings are neither sensitive nor specific. In addition it is an invasive procedure technique that should be probably considered only when other tests are inconclusive. In these patients nerve biopsy may occasionally identify vasculitis, amyloidosis, sarcoidosis, or tumor infiltration of the nerve [9].

\section{1.b Treatment and Management}

Several controlled studies have shown the efficacy of steroids [10], plasma exchange [11, 12], and IVIg in CIDP [13-17] with approximately 50-70\% of the patients responding to each of these treatments. In addition almost $50 \%$ of patients not responding to one of these treatments respond to the second therapy used leading to an improvement in $80 \%$ of reported patients $[18,19]$. The efficacy of these therapies was confirmed in recent Cochrane Reviews [20-23], in the Guidelines of the EFNS/PNS [9], and, for IVIg, in an evidence-based guideline of the American Academy of Neurology [24].

How to start treatment in CIDP: IVIg or steroids? It is often difficult for the clinician to decide what therapy should be first used in CIDP. This decision should consider the efficacy, cost and side effects of each these therapies. A few randomized trial have shown a comparable 
short-term efficacy of IVIg and oral corticosteroids [25] and of IVIg and plasma exchange [26] in CIDP and more recent trials have shown that both IVIg [13] and steroids [27] have prolonged efficacy in CIDP. A randomized controlled trial (IMC study) comparing the efficacy of sixmonth therapy with IVIg or intravenous methylprednisolone (IVMP) showed however, that IVIg were more frequently effective than steroids during the first six month of treatment [28]. This difference might have reflected however a more rapid efficacy of IVIg compared to steroids since patients not responding within two months to the first therapy were shifted to the alternative therapy. This hypothesis is supported by the results of the PREDICT study [29] where the median time to improvement in patients treated with pulsed monthly oral dexamethasone was 17 weeks and of 39 weeks for daily oral prednisolone while in the ICE trial, IVIg showed to be effective in most patients within the first two months of therapy [30]. In the IMC study it was also shown that, when effective, steroids were less frequently associated with deterioration after therapy discontinuation than IVIg [28]. This data were confirmed in the follow-up extension of the study [31] showing that a similar proportion of patients eventually deteriorated after discontinuing IVIg (87\%) or IVMP (79\%) even if the median time to deterioration was longer after discontinuing IVMP (14 months) than IVIg (4.5 months). A similar difference also derives from the PREDICT study where the median time to relapse after therapy discontinuation was 17.5 months after pulsed dexamethasone and 11 months after oral prednisolone [31] while in the ICE trial 45\% of the patients relapsed within 6 months after suspending 6 months therapy with IVIg [28]. A similar difference on the prolonged efficacy of the two treatment derives from a large retrospective study on 70 patients showing that the possibility to stop treatment tended to be more frequent in patients who responded to steroids than to IVIg [32]. 
Steroid are usually well tolerated over the short term even if agitation and hyperglycemia may early occur. Most common side effects over the long-term include hyperglycemia, hypertension, gastritis and gastrointestinal bleeding, osteoporosis, glaucoma, cataracts, poor wound-healing, susceptibility to infection, weight gain, depression, psychosis and insomnia. Therapy with IVIg is usually better tolerated, even if fever, flu like-syndrome and headache are not so uncommon. Serious adverse effects are rare and include tromboembolia, renal failure (mainly in patients with pre-existing renal failure), anaphylaxis (especially in patients with IgA deficiency) or aseptic meningitis. In the IMC trial [28] there were not significant differences in the proportion of patients experiencing adverse events even if slightly more adverse events were observed after steroids. The difference might become however more evident over the long period as shown in a retrospective study with a significantly higher proportion of patients experiencing adverse reaction after steroids (13\%) than after IVIg (4\%) [18]. A recent review of studies performed using daily or pulsed steroids showed however that pulsed monthly steroids are better tolerated than daily steroids with a consistent reduction in the frequency of most side effect [33]. One of the major inconvenience of IVIg therapy in CIDP is the fact that most patients require regular IVIg infusions to prevent relapses with maintenance dosage and frequency of infusion ranging from $0.5 \mathrm{~g} / \mathrm{kg}$ to $2 \mathrm{~g} / \mathrm{kg}$ every two to 6 weeks. The inconvenience of repeated IVIg infusions in CIDP can be solved with home infusion of subcutaneous immunoglobulin (SCIg) whose efficacy as maintenance treatment was show to be similar to IVIg in a small controlled study on 15 patients [34]. The follow-up extension of this study also confirmed the prolonged efficacy of SCIg [35]. A more recent study assessing the long-term efficacy of SCIg in CIDP and MMN, showed however that up to one third of the patients with CIDP required an increased dosage of SCIg or preferred to return to IVIg [36]. This data seems to be confirmed by the preliminary and yet unpublished data of a recent controlled trials that confirmed that SCIg is 
significantly more effective then placebo in preventing relapse within 6 months after suspending effective IVIg therapy even if one third of the patients relapsed within six months after passing from IVIg to SCIg [37]. This figure is higher than the $13 \%$ of relapse observed with IVIg over the same period in the extension phase of the ICE trial [13]. SCIg was recently shown to be similarly effective as IVIg as initial treatment in CIDP with only a modest delay in efficacy compared to IVIg [38]. The number of patients included in this study was however relatively small and the improvement in other parameters was quite marginal. SCIg remain a valid option for maintenance treatment in patients with CIDP reducing the inconvenience and cost of repeated hospital admission, when IVIg is not feasible at home, and the adverse events related to IVIg infusions. In addition, by performing the therapy at home patients do not loose time for their usual daily and working activities. Still the patients should be informed that up to one third of them may worsen when they pass from IVIg to SCIg.

The cost of therapy with IVIg (and of SCIg) is consistently higher than that of steroids [39, 40]. In Italy for instance the yearly cost of IVIg varies, depending on the weight of the patient and the maintenance dose used, from 30,000 to 80,000 Euro while that of oral or intravenous steroids does not reach 1,000 Euro. This may explain which IVIg therapy might be difficult to propose in low-income countries.

Plasma exchange. Even if considered as effective as IVIg or steroids in CIDP, plasma exchange is usually considered the third choice since it is more invasive for the patients and has a higher prevalence of side effects related to difficulty with venous access, use of citrate and hemodynamic changes [9]. In a retrospective study on 105 patients, a similar proportion of patients responded to plasma exchange (70\%) or IVIg (64\%) but more complications were observed after PE (10) than after IVIg (0) [41]. A similar difference in side-effects was observed in a retrospective study on 267 patients with CIDP treated with plasma exchange (19\%), steroids 
$(12,5 \%)$ or IVIg (4\%) [18]. Plasma exchange is rapidly effective as initial treatment in CIDP with improvement usually starting in a few days but the median time to deterioration after therapy discontinuation is relatively short with two-third of improved patients worsening within two weeks after stopping therapy [12]. These data together with the inconvenience related to the necessity of repeated admission for repeated exchanges makes of PE a less suitable option for the long-term treatment of CIDP [9]. It remains however a valid option for patients not responsive or becoming resistant to IVIg or steroids [18].

Immune suppressive or immune modulator agents. The necessity for long-term periodic treatments with IVIg or PE, the serious adverse events associated with the chronic use of steroids and the not so uncommon development of resistance to these therapies led in the recent years to an extensive use of immune suppressive or immune modulators agents in CIDP [42]. None of these therapies was shown to be effective in randomized controlled trials. Azathioprine given in addition to oral prednisolone for 9 months did not permit to reduce the dose of steroids [43]. A randomized study with oral methotrexate in addition to IVIg or steroids [44] showed that the therapy was well tolerated but was not more effective than placebo in reducing the dose of steroids or IVIg necessary to maintain the improvement. Similar negative results were obtained in two trials with intramuscular interferon beta-1a (IM IFN $\beta-1 a)[45,46]$ while a controlled trial with fingolimod was just interrupted after an interim analysis showing that the therapy was not effective [47].

Despite the negative results from randomized studies, immunosuppressive are still frequently used in patients with CIDP. This derives from the results of uncontrolled and often retrospective studies on small series of patients treated with other immune therapies including cyclosporine, interferon $\alpha$, mycophenolate mofetil, etanercept or tacrolimus (reviewed in [42]). The necessity to confirm these preliminary reports in controlled studies derives from the results 
of two controlled studies [44, 46] where $40 \%$ of the patients went into remission even under placebo. Not only a positive result may prompt the widespread use of a therapy whose efficacy might not be later confirmed, but also a negative data may block the use of a potentially useful therapy for several years. This might be the case of the lack of response in one patient to natalizumab [48] that was later reported to effective in three patients [49]. Some additional caution should be used with the data on stem cell transplantation [50] and alemtuzumab [51] whose efficacy in CIDP was often associated with severe complications. The need for caution with immune suppressive therapies in CIDP also derives from a retrospective study on 110 patients with CIDP not adequately responsive to IVIg, steroids or plasma exchange, where the proportion of patients responding to different immune suppressive therapies ranged between 20$30 \%$ while $10-20 \%$ of treated patients had adverse events related to the use of these therapies [52].

The therapeutic decision might be difficult for severely disabled patients not responding to conventional therapies. It is generally assumed that this is related to the development of axonal loss even if this aspect has not been clearly demonstrated. Few uncontrolled studies are consistent in showing that some disabled patients refractory to conventional therapies may respond to intravenous cyclophosphamide [53-55] while more recent studies showed the efficacy in some patients of rituximab [42] where improvement was mainly associated with the presence of an haematological disease and particularly of monoclonal gammopathy [56]. The possible beneficial effect of rituximab also derive from immunological studies showing that up to $10 \%$ of the patients with CIDP have IgG4 antibodies to the proteins at the node of Ranvier Contactin 1 or Neurofascin 155 [57-60]. These studies led to the opinion that in a minority patients the target for the immune response in CIDP may be the node and paranode (nodo-paranodopathy) affecting either axonal (such as contactin-1) or myelin (neurofascin-155) proteins. These patients were 
also reported to share a poor response to IVIg while some of them improved with rituximab [61]. This was related to the fact that IgG4 antibodies do not activate complement, so that the possible inhibitory effect of IVIg on complement activation did not affect the action of these antibodies. Even if the number of patients is still small, this finding support the hypothesis that CIDP may include different immune mediated neuropathies affecting the myelin, the node or paranode with different pathogenesis and response to therapy [2].

Treatment of atypical CIDP. A variable proportion of patients presents with an atypical form of CIDP including Lewis-Sumner syndrome, distal acquired demyelinating symmetric (DADS) neuropathy, purely motor, purely sensory and focal CIDP [7]. The proportion of patients with these atypical forms varies considerably in the literature from 1 to $50 \%$ [5, 7]. This might reflect the absence of definite diagnostic criteria for these atypical forms of CIDP and the fact that a variable proportion of patients with atypical CIDP at presentation eventually evolve into a typical CIDP. This may also explain the heterogeneity in the response to the therapy in these patients. It is for instance accepted that patients with pure motor CIDP have poor response and possibly worsen under therapy with steroids even if these conclusion derives from very few studies [62-64]. There are also difference in the response to therapy in patients with Lewis Sumner syndromes with a proportion of patients responding to IVIg ranging in different studies from $38 \%$ [65] to $80 \%$ [66] compared to $80 \%$ of those with typical CIDP. In one of these studies there was also a poor response to plasma exchange (17\%) compared to typical CIDP (81\%) [65]. All these discrepancies leave it unclear whether these atypical forms of CIDP are indeed variants of CIDP or whether they may represent different demyelinating neuropathies [2].

\section{1.c How we treat CIDP}


We prefer to delay the treatment in patients with mild sensory deficits and minimal or no weakness that are not limited or consistently disturbed in their daily life explaining to the patient that spontaneous improvement may occasionally occur and that the possible adverse events or inconvenience related to therapy may be even more disturbing than mild neuropathy symptoms. In patients who do not improve, continue to worsen, or have motor and sensory deficits at presentation or are disturbed by their sensory symptoms, treatment with steroids or IVIg is first considered after discussing with the patients the pro and cons of each treatment.

Contraindications to corticosteroids will influence the choice towards IVIg and vice versa. In patients with purely motor CIDP, we still consider IVIg as the first choice. If we need however to use corticosteroids, we closely monitor patients for possible deterioration. Patients not responding to IVIg or steroids are shifted to the other therapy. We should however remember that steroids may become effective after several weeks and that over $50 \%$ of the patients responsive to IVIg do so after the second course of IVIg. We prefer to reserve plasma exchange for patients not responsive to the other two therapies.

For IVIg we start at the dose of 2 gm/kg body weight over 3-5 consecutive days. The onset of action is rapid, and the side effect profile is rather benign. In a few patients, the effects of IVIg are sustained eliminating the need for additional treatment. When improvement is temporary, we give IVIg periodically starting with the dose of $1 \mathrm{~g} / \mathrm{kg}$ at a frequency sufficient to prevent the wear-off effect of therapy. The dosage of IVIg is subsequently slowly reduced to achieve the minimal effective dose. We consider subcutaneous immunoglobulin a valid alternative for chronic therapy with immunoglobulins as far as they are given at the same (or even higher) monthly dose IVIg.

For steroids, we prefer to use monthly course of intravenous methylprednisolone at the starting dose of 2g over 2-4 consecutive days. Pulsed oral dexamethasone (40 mg for four days) 
is a valid alternative. This pulsed regimen can be also performed in patients with diabetes or glucose intolerance in whom we prefer to admit patients to better monitor and adjust glucose levels with insulin. Daily oral steroids can be also used at the standard daily dosage of $60 \mathrm{mg} /$ day of prednisone even if this regimen may have more frequent adverse events than pulsed therapy. Patients should be educated regarding the side effects of steroids and instructed in the use of supplemental calcium and vitamin D, gastro protection and on the need for monitoring of blood pressure, glucose and electrolytes. After 2-3 months, the dose of steroid can be gradually reduced and eventually suspended after 6 months closely monitoring the possible worsening of the patients in which case the effective therapy is resumed. Daily oral steroids may be shifted to an alternate day regimen after 6-8 weeks that is better tolerated than the daily regimen.

In patients non responsive or having contraindications to IVIg and steroids we perform plasma exchange (usually 4-5 courses of one plasma volume on alternate days). The onset of action generally is rapid. If the effect is sustained, no further treatment is required. If improvement occurs but the patient relapses, plasma exchange is initially repeated after one or two weeks and subsequently delayed to a frequency of 1 course every 1-2 months.

Due to lack of consistent evidence supporting the use of immune suppressive agents we reserve them to patients who remain disabled despite the above mentioned therapies or who have failed to respond to all of them, while we do not to use them to reduce the cost of therapy. In these patients we use intravenous cyclophosphamide a the monthly dose of $0.7-1 \mathrm{~g} / \mathrm{m}^{2}$ for six months. In patients with an associated monoclonal gammopathy or in those not improving after cyclophosphamide we use rituximab at the dose of $1 \mathrm{~g}$ repeated after two weeks.

\section{MULTIFOCAL MOTOR NEUROPATHY}

\section{2.a Clinical features and diagnosis}


Multifocal motor neuropathy (MMN) is a purely motor mono neuritis multiplex characterized by the presence of multifocal partial conduction blocks (CB) on motor nerves [67, 68] .The frequent association of MMN with antibodies to the ganglioside GM1 and the improvement observed in most patients after IVIg support the opinion that the disease is immunologically mediated. MMN is a rare neuropathy with a prevalence of $0.5-0.6$ per 100,000 inhabitants [67, 68]. MMN almost invariably presents with progressive, usually distal, asymmetric weakness in the upper limbs in the distribution of individual and usually not contiguous motor nerves $[67,68]$. One of the typical feature of this neuropathy is the absence of sensory impairment even in the territory of affected sensorimotor nerve. Some patients may report mild sensory symptoms but only a minority of them have a minor sensory loss. Most patients MMN carries an overall good prognosis quoad vitam, even if the majority of them become disabled in their daily life because of a reduced dexterity in manual activities, while few patients become disabled in walking [67].

Diagnostic criteria for MMN have been proposed by several groups including the American Association of Neuromuscular and Electrodiagnostic Medicine (AANEM)[70] and the Joint Task force of the EFNS/PNS [71]. The diagnosis mainly relies on the presence of persistent, multifocal, partial CB in motor nerves outside the usual sites of nerve compression. The diagnosis is also supported by the frequent occurrence of increased levels of serum IgM antibodies to the ganglioside GM1 with frequency in most laboratory of $40-50 \%$ [67]. These antibodies are not specific for this disease and can be also found in other immune neuropathies and in 5-10\% of patients with MND even if the majority of them have lower antibody levels than patients with MMN. Several attempts have been performed to improve the sensitivity of anti-GM1-antibodies in MMN using more sophisticated techniques or testing GM1 in addition to other glycolipids. The combination of GM1 with galactocerebroside increases the sensitivity of 
GM1 testing in MMN of approximately 20\% with a marginal reduction of the specificity and positive predictive value for MMN [72]. Magnetic resonance (MR) imaging studies of the forearm or brachial plexus may show in some patients an asymmetrically increased signal intensity in T2 -weighted images or in T1-weighted images after gadolinium enhancement associated with diffuse nerve swelling of the brachial plexus [73]. Similarly promising results were observed with ultrasound study of the nerves [74].

\section{2.b Treatment and management}

Almost $80 \%$ of patients with MMN respond to IVIg [75], whose efficacy has been confirmed in five randomized, double-blind, placebo-controlled trials on a total of 75 patients [76-80]. IVIg induces a rapid improvement which often occurs within one week of treatment and is usually more evident in recently affected regions with minor or no effect on stabilized deficits. Only a few patients have persistent improvement after a single or few courses of therapy while in most patients the effect of IVIg lasts a few weeks and has to be maintained with periodic IVIg infusions for long periods of time [81-84]. Maintenance therapy can be also performed with subcutaneous immunoglobulin (SCIg) whose efficacy as maintenance treatment has been confirmed in two small randomized controlled trials [86, 87]. A recent study assessing the longterm efficacy of SCIg in MMN, showed however that almost 50\% of the patients with MMN required an increased dosage of SCIg or preferred to return to IVIg [36].

A minority of patients with MMN do not respond or become resistant to IVIg while in others IVIg may be difficult to perform because of their elevated cost. Steroids and plasma exchange are ineffective in MMN and can be also associated with clinical worsening [67]. Highdose intravenous cyclophosphamide, oral azathioprine and interferon $-\beta 1$ a (IFN- $\beta 1 \mathrm{a}$ ) were also reported to be effective in some patients in open uncontrolled studies [88] while the only 
randomized double blind controlled trial with immunosuppressant in MMN showed that mycophenolate mofetil did not permit to increase the effectiveness or to reduce the dose of IVIg [89]. More recent open trials showed that oral methotrexate [90] and eculizumab [91] were marginally effective in some patients but did not permit to consistently reduce the dose of IVIg therapy. A positive effect was also reported with rituximab [89] even if the results were not subsequently confirmed [93]. A recent Cochrane review concluded that at the present time there is little evidence to support the use of any immunosuppressant in MMN [88], and confirmed that IVIg remains the gold standard for the treatment of MMN.

\section{2.c How we treat MMN}

In MMN we start with IVIg therapy at the standard dose of $2 \mathrm{~g} / \mathrm{kg}$ on 2-5 consecutive days followed by maintenance infusions ranging from $0.4 \mathrm{~g} / \mathrm{kg}$ once a week to $1-2 \mathrm{~g} / \mathrm{kg}$ every $2-5$ weeks. We frequently adjust however therapy in these patients since over time, several patients become progressively less responsive to IVIg and require increasing dosage (in case of insufficient response) or frequency (in case of reduced duration of the response) of IVIg to maintain improvement. SCIg is a valid alternative for maintenance treatment even if the dosage often needs to be increased over time. In the few patients not responding or becoming resistant to IVIg we consider the use of use intravenous cyclophosphamide a the monthly dose of 0.7$1 \mathrm{~g} / \mathrm{m} 2$ for six months.

\section{NEUROPATHY ASSCIATED WITH IgM MONOCLONAL GAMMOPATHY AND ANTI-MAG ANTIBODIES}

3.a Clinical features and diagnosis 
Almost 50\% of patients with neuropathy associated with IgM monoclonal gammopathy have antibodies to the myelin-associated glycoprotein (MAG) $[94,95]$. This is a rare neuropathy with an estimated prevalence of at least 20 per 100.000 in the population above 50 years [94]. The majority of affected patients are men presenting their first neuropathy symptoms in the sixth or seventh decade. Almost $80 \%$ of them have IgM MGUS while most remaining patients have Waldenström’s macroglobulinemia (WM) that in these patients is frequently indolent.

Most patients present with sensory symptoms in legs including distal paresthesias, hypodysestesia, cramps or other pains and unsteadiness of gait. Weakness is frequently absent at onset. An intentional and postural tremor in the upper limbs is often reported being sometime quite disabling and in some patients it can be the presenting symptom. On neurological exhamination, the neuropathy is characterized by a distal and symmetric, predominantly deep sensory involvement, gait ataxia and postural tremor in the upper limbs while motor impairment is usually less prominent and often appears later. The majority of patients with anti-MAG antibodies have a relatively favourable long-term prognosis with $25-30 \%$ of them becoming at least moderately disabled after 10 year and 50\% after 15 years [96, 97].

\section{3.b Treatment and management}

Several data support the possible pathogenetic role of anti-MAG IgM in the neuropathy [94] as also confirmed by the correlation of clinical improvement with the reduction of antibody titers. Almost 50\% of reported patients improved, at least temporarily, after one of more immune therapies including steroids, plasmaexchange, a number of cytotoxic agents, IVIg, fludarabine, cladribine and interferon- $\alpha$ [96]. The efficacy of of these therapies has not been so far confirmed in randomised controlled trials most of whom showed at the most a marginal effect [98]. More recently a number of open pilot trials have suggested the efficacy in these patients of the 
humanised monoclonal antibody rituximab directed against the CD20 antigen [99-102]. The efficacy of rituximab in anti-MAG neuropathy was also assessed in two controlled trials, one on 26 patients [103] and the other on 54 patients [104]. Even if neither trial met the pre-specified primary outcome measures, both studies showed a $20 \%$ to $30 \%$ absolute increase in the number of patients treated with rituximab improving in disability compared to those receiving placebo. A recent revision combining the results of these two trials concluded that rituximab is beneficial in this neuropathy improving disability and the subjective impression of change [98]. In responding patients the benefit was reported to last up to 2 years in $80 \%$ of the patients and 3 years in $60 \%$ of them [105]. This aspect is important for the long term management of the patients and should be balanced with the side effects including the occasional development of progressive multifocal leucoencephalopathy [106]. Recent studies on small series of patients and the revision of a large series of french patients reported on the possible additional efficacy of the association of rituximab with other therapy used in WM including fludarabine [107] and bendamustine [108]. These results should be however confirmed in controlled studies. Even if plasma exchange was not shown to be more effective than placebo when given in addition to immune therapies, it may be useful in some patients with a rapidly progressive course [109]. Some caution in the interpretation of these results derives however from the fact that variable levels of anti-MAG antibodies were considered to be diagnostic for this neuropathy. In our laboratory for instance antibodies lower that $1 / 6,400$ by western blot assay with purified human myelin were no strictly associated with this neuropathy [110]. A more recent commercially available ELISA system considered abnormal values above 1,000 Bühlmann titer. Even if this system has the advantage to avoid the cumbersome procedure of home-made assays, it carries the risk of including a more heterogeneous group of patients [110] highlighting the 
necessity to better define cut-off levels for the definition of this neuropathy based on adequately large control population.

\section{3.b How we treat neuropathy associated with anti-MAG antibodies}

We curently use symptomatic therapies for tremor and paresthesias in symptomatic patients with neuropathy asssociated with anti-MAG antibodies who are not impaired in daily acivities and reassure them on the usually favourable functional prognosis for several years. In patients impaired in their daily-life or who have evidence of an unusually rapid progression we consider rituximab as the first option at the dose of 375mg/m2 every weeks for four weeks. We eventually repeat this dose after six months if the first course did not stop the progression of symptoms especially if there is an insufficinet reduction of B-cell counts while in patients stabilized or improved we consider an extra infusion after 6 and 12 months to prevent a rebound of B-cells. We occasionally propose a course of plasma exchange in patients with an unusal rapid progression of the neuropathy to reduce the progression of the neuropathy or in patients not responding to rituximab.

\section{CONCLUSION}

In the last twenty years several studies have permitted to demonstrate the efficacy of a number of immune therapies in immune mediated neuropathies and to confirm that these neuropathies, even if all characterized by a pathological process involving nerve myelin or the node of Ranvier, are separate clinical entities with different responses to therapies. Even within CIDP, the possibility that this is not a disease with different clinical presentation but a syndrome including different clinical entities with different pathogenesis and response to therapies is currently extensively investigated. 
From all these studies it also appear that beside the efficacy of IVIg, steroids and PE in CIDP, IVIg in MMN and possibly rituximab in neuropathy associated with anti-MAG antibodies, there is inconsistent evidence from controlled studies that the other immune therapies currently used to reduce the frequency of IVIg or PE or the adverse events frequently associated with the chronic use of steroids, may have efficacy in these neuropathies. In addition most of these studies showed that a similar proportion of patients treated with these therapies worsen after the suspenison of the effective therapy compared to patients treated with placebo. This fact highlight the need for controlled studies in these neuropathies to ensure that the frequently reported improvement in uncontrolled studies reflect their real efficay and not a sponatenaous stabilization or remission of the disease.

\section{Expert commentary}

Ther are still unanswered problems in the treatment of these neuropathies. In CIDP it is unclear whether the more prolonged efficay of steroids may balance the higher cost and better tolerability of IVIg. It is also unclear whether the suggested combined inital use of IVIg and pulsed steroids may unify the advantage of the more rapid efficay of IVIg with the prolonged effiacy of steroids. A new randomized controlled trial comparing the efficacy of IVIg with that of IVIg combined with steroids is under consideration. Despite their widespraed use, there is no data from ramdomized controlled trial supporting the efficay of immune suppressive therapy in CIDP. I think that particular attention should be given to the newly developed monoclonal antibodies including ocrelizumab, alemtuzumab and natalizumab that were shown to be effective in patients with multiple sclerosis (MS), even if the results obtained in immune mediated neuropathies with therapies effective in MS have been so far quite disappointing. Further studies 
on the possible pathogenetic mechanism of CIDP are also needed to clarify whetere the presence of different antibody reactivities in these patients may reflect the presence of different clinical entities and whether these may have different response to therapies.

Things are possibly more simple in MMN where there is not much choice in the initial treatment of the disease. Most stsudies are now addressing the possible benefit in these patients (as in those with CIDP) of the use of SCIg as maintenace treatment. This will not really change the therapeutical strategy in these patients but might solve some of the incovenience related to the need of chronic IVIg infusions. It remain however unclear why in this disease, despite the considerable evidence supporting an autoimmune pathogenesis, other immune therapies including plasma exchane and steroids are not effective and may even worsen the disease. A similar lack of evidence of the effiacy of any other immune therapies in MMN also support the need to better understand what is the effective pathogenesis of MMN and whether there are other possible mechamisms of action of IVIg that might reveal a possible non immune mediated action of this therapy in MMN.

Even if several data suppot the hypothesis that anti-MAG antibodies have a role in causing the neuropathy, this neuropathy does not really represent a typical autoimmune disease considering the slowly progressive course of the disease, the absence of temporal variability in antibody leves and their constant monoclonality. This is why most of the therapies used in this neuropathy are those used in hematological malignacy with sparse data on their long-term efficay and safety. Most studies in patients with anti-MAG neuropathy report indeed the effiacy of these therapies for no more than two years while the disease lasts in most patients several years. Better data are therfore needed to clarify what would be the best regimen and therapy to be used in this neuropathy over the long term. 


\section{FIVE-YEAR VIEW}

The widespread interest in these immune neuropathies derives form the fact that these are among the best treatable chronic neuropathies. This has led to the performance of several controlled studies in this field in the last twenty years. Despite the demonstration in a few studies of the efficay of IVIg in CIDP and MMN, regular authorities have so far decided that not all brands of IVIg should be registerd for their use in these neuropathies unless their proven efficacy even if recent observational data support their similar efficacy in these neuropathies [110]. This has led to a number of registrative studies with different brand of IVIg and possibly of SCIg diverting the attention of clinicians to these usually remunerative studies. This also because studies with other expensive therapies might be difficult without the support of producing companies that have a limited interested in these rare neuropathies. All this risks to slow in the next few years the study of new therapies in these neuropathies leaving the patients dependent on the chronic use of immunoglobulin with the only possible choice between their intravenous or subcutaneous use. 


\section{KEY ISSUES}

- Chronic inflammatory neuropathies are an heterogeneous group of rare disorders caused by an autoimmune reaction against peripheral nerve. Several studies have shown the efficacy of immune therapies in these neuropathies and confirm that these neuropathies, even if all characterized by a pathological process involving nerve myelin or the node of Ranvier, are separate clinical entities with different responses to therapies.

- IVIg are more frequently or rapidly effective than steroids in CIDP even if steroids, when effective, are less frequently associated with deterioration after therapy discontinuation. PE is also effective in CIDP but it is less used as it is more invasive and has more side effects. SCIg are effective as maintenance therapy in CIDP while the efficacy of immune suppressive therapies has not been so far confirmed in controlled studies.

- IVIg remain the only therapy of proven efficacy in MMN. Its efficacy needs however to be maintained with periodic IVIg infusions or with SCIg. There are no data from controlled studies supporting the efficacy of immune suppressive therapies in MMN.

- $\quad$ Recent controlled studies have shown that rituximab its effective in neuropathy associated with anti-MAG antibodies even if its efficacy and safety over the long-term are still unsettled. A better definition of cut-off levels for antibody levels in this neuropathy should be however defined. 


\section{References}

1. Vallat J-M, Sommer C, Magy L. Chronic inflammatory demyelinating polyradiculoneuropathy: diagnostic and therapeutic challenges for a treatable condition. Lancet Neurol. 2010;9:402-12.

2. Nobile-Orazio E. Chronic Inflammatory demyelinating polyradiculoneuropathy. Where we are, where we should go. J Peripher Nerv Syst. 2014;19:2-13.

3. Lunn MPT, Manji H, Choudhary PP, et al. Chronic inflammatory demyelinating polyradiculoneuropathy: a prevalence study in south east England. J Neurol Neurosurg Psychiatry. 1999;66:677-680.

4. Mahdi-Rogers M, Hughes RAC. Epidemiology of chronic inflammatory neuropathies in south-east England. Eur J Neurol. 2014;21(1):28-33.

5. Lefter S, Hardiman O, Ryan AM. A population-based epidemiologic study of adult neuromuscular disease in the Republic of Ireland. Neurology.2017;88:304-313

6. Hughes RAC, Allen D, Makowska A, Gregson NA. Pathogenesis of chronic inflammatory demyelinating polyradiculoneuropathy. J Peripher Nerv Syst. 2006;11:30-46.

7. ** Mathey EK, Park SB, Hughes RA, et al. Chronic inflammatory demyelinating polyradiculoneuropathy: from pathology to phenotype J Neurol Neurosurg Psychiatry. 2015;86:973-85.

In this review the authors report some of the more recent issues in the diagnosis of CICDP and its variants and summarize recent advances on the immune pathogenesis of CIDP

8. * Beriner A, BrannaganTH, Comparison of sensitivity and specificity among 15 criteria for chronic inflammatory demyelinating polyneuropathy. Muscle Nerve. 2014;50(1):40-6. 
An useful review of the principle diagnostic criteria used in CIDP with a comparison on their sensitivity and specificity

9. ** Joint Task Force of the EFNS and the PNS. European Federation of Neurological Societies/Peripheral Nerve Society Guideline on management of chronic inflammatory demyelinating polyradiculoneuropathy: report of a joint task force of the European Federation of Neurological Societies and the Peripheral Nerve Society-First Revision. J Peripher Nerv Syst. 2010;15:1-9. Erratum in: J Peripher Nerv Syst. 2010;15:373. This is an historical and outstanding guidelines on the diagnosis and treatment of CIDP. All recent randomized controlled studies now use this guideline for the inclusion of patients in trials.

10. Dyck PJ, O’Brien PC, Oviatt KF, et al. Prednisone improves chronic inflammatory demyelinating polyradiculoneuropathy more than no treatment. Ann Neurol. 1982;11:136144.

11. Dyck PJ, Daube J, O’Brien P, et al. Plasma exchange in chronic inflammatory demyelinating polyradiculoneuropathy. N Engl J Med. 1986;314:461-465.

12. Hahn AF, Bolton CF, Pillay N, et al. Plasma-exchange therapy in chronic inflammatory demyelinating polyneuropathy: a double-blind, sham-controlled, cross-over study. Brain 1996;119:1055-1066.

13. ** Hughes RA, Donofrio P, Bril V, et al. Intravenous immune globulin (10\% caprylatechromatography purified) for the treatment of chronic inflammatory demyelinating polyradiculoneuropathy (ICE study): a randomized placebo-controlled trial. Lancet Neurol. 2008;7:136-44.

This landmark study first proved the efficacy of IVIg in a randomized controlled study in CIDP. 
14. Vermeulen M, van Doorn PA, Brand A, et al. Intravenous immunoglobulin treatment in patients with in chronic inflammatory demyelinating polyneuropathy: a double-blind, placebo controlled study. J Neurol Neurosug Psychiatry.1993;56: 36-39

15. Thompson N, Choudhary P, Hughes RAC, et al. A novel trial design to study the effect of intravenous immunoglobulin in chronic inflammatory demyelinating polyradiculoneuropathy. J Neurol.1996;243:280-285.

16. Hahn AF, Bolton CF, Zochodne D, et al. Intravenous immunoglobulin treatment in chronic inflammatory demyelinating polyneuropathy: A double blind, placebo-controlled, cross-over study. Brain. 1996;119:1067-1077.

17. Mendell JR, Barohn S, Freimer ML, et al. Randomized controlled trial of IVIg in untreated chronic inflammatory demyelinating polyradiculoneuropathy. Neurology. 2001;56:445-449.

18. Cocito D, Paolasso I, Antonini G, et al. A nationwide retrospective analysis on the effect of immune therapies in patients with chronic inflammatory demyelinating polyradiculoneuropathy. Eur J Neurol. 2010;17:289-294.

19. Viala K, Maisonobe T, Stojkovic T, et al. A current view of the diagnosis, clinical variants, response to treatment and prognosis in chronic inflammatory demyelinating polyradiculoneuropathy. J Peripher Nerv Syst. 2010;15:50-56.

20. * Mehndiratta MM, Hughes RAC. Corticosteroids for chronic inflammatory demyelinating polyradiculoneuropathy. Cochrane Database Syst Rev. 2015;1:CD002062.

This complete and updated review analyze all the studies with steroids in CIDP

21. * Mehndiratta MM, Hughes RAC, Agarwal P. Plasma exchange for chronic inflammatory demyelinating polyradiculoneuropathy. Cochrane Database Syst Rev. 2012;9:CD003906. This complete and updated review analyze all the studies with plasma exchange in CIDP 
22. * Eftimov F, Winer JB, Vermeulen M, et al. Intravenous immunoglobulin for chronic inflammatory demyelinating polyradiculoneuropathy. Cochrane Database Syst Rev. 2013;12:CD001797.

This complete and updated review analyze all the studies with IVIg in CIDP

23. ** Oaklander AL, Lunn MPT, Hughes RAC, et al. Treatments for chronic inflammatory demyelinating polyradiculoneuropathy (CIDP): an overview of systematic reviews (Review) Cochrane Database of Systematic Reviews 2017;1: CD010369.

This very recent review summarizes and compares the data from all previous reviews on the treatment of CIDP

24. Patwa HS, Chaudhry V, Katzberg H, et al. Evidence-based guideline: Intravenous immunoglobulin in the treatment of neuromuscular disorders. Report of the Therapeutics and Technology Assessment Subcommittee of the American Academy of Neurology. Neurology. 2012;78:1009-1015

25. Hughes RAC, Bensa S, Willison HJ, et al. Randomized controlled trial of intravenous immunoglobulin versus oral prednisolone in chronic inflammatory demyelinating polyradiculoneuropathy. Ann Neurol. 2001;50:195-201.

26. Dyck PJ, Litchy WJ, Kratz KM, et al. A plasma exchange versus immune globulin infusion trial in chronic inflammatory demyelinating polyradiculoneuropathy. Ann Neurol. 1994;36:838-845.

27. Eftimov F, Vermeulen M, van Doorn PA, et al. Long-term remission of CIDP after pulsed high-dose dexamethasone or short term prednisolone treatment. Neurology. 2012;78:107984. 
28. ** Nobile-Orazio E, Cocito D, Jann S, et al. Intravenous immunoglobulin versus intravenous methylprednisolone for chronic inflammatory demyelinating polyradiculoneuropathy: a randomised controlled trial. Lancet Neurol. 2012;11:493-502.

This controlled study compare over the prolonged efficacy of IVIg and intravenous steroids in CIDP showing that IVIg are more frequently effective while steroids, when effective, are associated with a more prolonged efficacy

29. van Schaik IN, Eftimov F, van Doorn PA, et al. Pulsed high-dose dexamethasone versus standard prednisolone treatment for chronic inflammatory demyelinating polyradiculoneuropathy (PREDICT study): a double-blind randomised controlled trial. Lancet Neurol. 2010; 9:245-253.

30. Latov N, Deng C, Dalakas MC, et al. Timing and Course of Clinical Response to Intravenous Immunoglobulin in Chronic Inflammatory Demyelinating Polyradiculoneuropathy. Arch Neurol. 2010;67(7):802-807.

31. * Nobile-Orazio E, Cocito D, Jann S, et al. Frequency and time to relapse after discontinuing 6-month therapy with IVIg or pulsed methylprednisolone in CIDP. J Neurol Neurosurg Psychiatry. 2015;86:729-34.

This study confirm that both IVIg and steroids do not cure CIDP but confirm that the time to deterioration after therapy discontinuation is significantly longer after steroid than IVIg therapy.

32. Rabin M, Mutlu G, Stojkovic T, et al. Chronic inflammatory demyelinating polyradiculoneuropathy : search for factors associated with treatment dependence or successful withdrawal. J Neurol Neurosurg Psychiatry. 2014;85:901-6 
33. Press R, Hiew FL, Rajabally YA. Steroids for chronic inflammatory demyelinating polyradiculoneuropathy: evidence base and clinical practice. Acta Neurol Scand. 2016;133:228-38.

34. * Markvardsen LH, Debost JC, Harbo T, et al. Subcutaneous immunoglobulin in responders to intravenous therapy with chronic inflammatory demyelinating polyradiculoneuropathy. Eur J Neurol. 2013;20(5):836-42.

This small randomized controlled study confirm the efficacy of SCIg as maintenance treatment in CIDP.

35. Markvardsen LH, Harbo T, Sindrup SH, et al. Subcutaneous immunoglobulin preserves muscle strength in chronic inflammatory demyelinating polyneuropathy. Eur J Neurol. 2014;21:1465-1470.

36. Cocito D, Merola A, Romagnolo A, et al. Subcutaneous immunoglobulin in CIDP and MMN: a different long-term clinical response? J Neurol Neurosurg Psychiatry. 2016;87(7):791-3.

37. [unpublished data; they will be presented at the AAN meeting in Boston, April 2017]

38. Markvardsen LH, Sindrup SH, Christiansen I, et al. Subcutaneous immunoglobulin as firstline therapy in treatment-naive patients with chronic inflammatory demyelinating polyneuropathy: randomized controlled trial study Eur J Neurol. 2017;24:412-418.

39. McCrone P, Chisholm D, Knapp M, et al. Cost-Utility Analysis of Intravenous Immunoglobulin and Prednisolone for Chronic Inflammatory Demyelinating Polyradiculoneuropathy (CIDP). Eur J Neurol. 2003;10:687-694.

40. Mahdi-Rogers M, McCrone P, Hughes RA. Economic costs and quality of life in chronic inflammatory neuropathies in southeast England. Eur J Neurol. 2014;21(1):34-9. 
41. Choudhary PP, Hughes RA. Long-term treatment of chronic inflammatory demyelinating polyradiculoneuropathy with plasma exchange or intravenous immunoglobulin. QJM. 1995;88: 493-502.

42. * Mahdi-Rogers M, van Doorn PA, Hughes RAC. Immunomodulatory treatment other than corticosteroids, immunoglobulin and plasma exchange for chronic inflammatory demyelinating polyradiculoneuropathy. Cochrane Database Syst Rev. 2013;6:CD003280. This complete and updated review analyze all the studies with immune suppressive therapy in CIDP

43. Dyck PJ, O’Brien P, Swanson C, et al. Combined azathioprine and prednisone in chronic inflammatory demyelinating polyneuropathy. Neurology. 1985; 35:1173-76.

44. RMC Trial Group. Pilot Randomised Controlled Trial of Methotrexate for Chronic Inflammatory Demyelinating Polyradiculoneuropathy (RMC Trial). Lancet Neurol. 2009;8:158-164

45. Hughes RAC, Gorson KC, Cros D, et al. Intramusculatr interferon beta-1a in chronic inflammatory demyelinating polyradiculoneuropathy. Neurology. 2010;74:651-657.

46. Hadden RDM, Sharrack B, Bensa S, et al. Randomized trial of interferon $\beta-1 \mathrm{a}$ in chronic inflammatory demyelinating polyradiculoneuropathy. Neurology. 1999;53:57-61.

47. Hartung HP, Dalakas M, Merkies I, et al. Oral Fingolimod in Chronic Inflammatory Demyelinating Polyradiculoneuropathy (FORCIDP): Results from a Phase III Randomized Placebo-controlled Trial. American Academy of Neurology 69th Annual Meeting, April 2228, 2017, Boston, MA (abstract).

48. Wolf C, Menge T, Stenner MP, et al. Natalizumab treatment in a patient with chronic inflammatory demyelinating polyneuropathy. Arch Neurol. 2010;67:881-3. 
49. Vallat JM, Mathis S, Ghorab K, et al. Natalizumab as a Disease-Modifying Therapy in Chronic Inflammatory Demyelinating Polyneuropathy - A Report of Three Cases. Eur Neurol. 2015;73:294-302.

50. Press R, Askmark H, Svenningsson A, et al. Autologous haematopoietic stem cell transplantation: a viable treatment option for CIDP. J Neurol Neurosurg Psychiatry. 2014;85:618-24.

51. Marsh EA, Hirst CL, Llewelyn JG, et al. Alemtuzumab in the treatment of IVIG-dependent chronic inflammatory demyelinating polyneuropathy J Neurol. 2010;257(6):913-9.

52. Cocito D, Grimaldi S, Paolasso I, et al. Immunosuppressive treatment in refractory chronic inflammatory demyelinating polyradiculoneuropathy. A nationwide retrospective analysis. Eur J Neurol. 2011;18:1417-21.

53. Good JL, Chehrenama M, Mayer RF, et al. Pulse cyclophosphamide therapy in chronic inflammatory demyelinating polyneuropathy. Neurology. 1998;51:1735-1738.

54. Brannagan TH 3rd, Pradhan A, Heiman-Patterson T, et al. High-dose cyclophosphamide without stem-cell rescue for refractory CIDP. Neurology. 2002;58:1856-8.

55. Gladstone DE, Prestrud AA, Brannagan TH 3rd. High-dose cyclophosphamide results in long-term disease remission with restoration of a normal quality of life in patients with severe refractory chronic inflammatory demyelinating polyneuropathy. J Peripher Nerv Syst. 2005;10:11-6.

56. Benedetti L, Briani C, Fazio R, et al. Rituximab in patients with CIDP: A report of 13 cases and review of the literature. J Neurol Neurosurg Psychiatry. 2011;82:306-8.

57. Querol L, Nogales-Gadea G, Rojas-Garcia R, et al. Antibody to contactin-1 in chronic inflammatory demyelinating polyneuropathy. Ann Neurol. 2013;73:370-380. 
58. Querol L, Nogales-Gadea G, Rojas-Garcia R, et al. Neurofascin IgG4 antibodies in CIDP associate with disabling tremor and poor response to IVIg. Neurology. 2014; 82:879-86.

59. Miura Y, Devaux JJ, Fukami Y, et al. Contactin 1 IgG4 associates to chronic inflammatory demyelinating polyneuropathy with sensory ataxia. Brain. 2015;138:1484-91.

60. Devaux JJ, Miura Y, Fukami Y, et al. Neurofascin-155 IgG4 in chronic inflammatory demyelinating polyneuropathy. Neurology. 2016; 86:800-7

61. * Querol L, Rojas-García R, Diaz-Manera J, et al. Rituximab in treatment-resistant CIDP with antibodies against paranodal proteins. Neurol Neuroimmunol Neuroinflamm 2015;2(5):e149.

This study first highlights the fact that different antibody reactivities may occur in CIDP and that their presence may predict a different response to therapy

62. Sabatelli M, Madia F, Mignogna T, et al. Pure motor chronic inflammatory demyelinating polyneuropathy. J Neurol. 2001;248:772-7.

63. Donaghy M, Mills KR, Boniface SJ, et al. Pure motor demyelinating neuropathy: deterioration after steroid treatment and improvement with intravenous immunoglobulin. J Neurol Neurosurg Psychiatry. 1994;57:778-783

64. Kimura A, Sakurai T, Koumura A, et al. Motor-dominant chronic inflammatory demyelinating polyneuropathy. J Neurol. 2010;257:621-9.

65. Kuwabara S, Isose S, Mori M, et al. Different electrophysiological profiles and treatment response in 'typical' and 'atypical' chronic inflammatory demyelinating polyneuropathy. J Neurol Neurosurg Psychiatry. 2014;86:1054-9.

66. Tackenberg B, Lünemann JD, Steinbrecher A, et al. Classifications and treatment responses in chronic immune-mediated demyelinating polyneuropathy. Neurology. 2007;68:1622-9. 
67. Nobile-Orazio E, Cappellari A, Priori A. Multifocal Motor Neuropathy: current concepts and controversies. Muscle Nerve. 2005;31:663-680.

68. Van Asseldonk JT, Franssen H, Van den Berg-Vos RM, et al. Multifocal motor neuropathy. Lancet Neurol. 2005;4:309-19.

69. Cats EA, van der Pol WL, Piepers S, et al. Correlates of outcome and response to IVIg in 88 patients with multifocal motor neuropathy. Neurology. 2010;75:818-25.

70. Olney RK, Lewis RA, Putnam TD, et al. Consensus criteria for the diagnosis of multifocal motor neuropathy. Muscle Nerve. 2003;27:117-121.

71. ** Joint Task Force of the EFNS and the PNS. European Federation of Neurological Societies/ Peripheral Nerve Society Guideline on management of multifocal motor neuropathy. Report of a joint task force of the European Federation of Neurological Societies and the Peripheral Nerve Society. First revision. J Peripher Nerv Syst. 2010;15:295-301.

This outstanding guideline on the diagnosis and treatment of MMN is the baseline for therapeutic studies $M M N$.

72. Nobile-Orazio E, Giannotta C, Musset L, et al. Sensitivity and predictive value of antiGM1/galactocerebroside IgM antibodies in multifocal motor neuropathy. J Neurol Neurosurg Psychiatry. 2014;85:754-758.

73. Van Es HW, Van den Berg LH, Franssen H, et al. Magnetic resonance imaging of the brachial plexus in patients with multifocal motor neuropathy. Neurology. 1997;48:1218-4.

74. Kerasnoudis A, Pitarokoili K, Behrendt V, et al. Multifocal motor neuropathy: correlation of nerve ultrasound, electrophysiological, and clinical findings. J Peripher Nerv Sys. 2014;19:165-174 
75. * van Schaik IN, van den Berg LH, de Haan R, et al. Intravenous immunoglobulin for multifocal motor neuropathy. Cochrane Database Syst Rev. 2005;2:CD004429. This is a quite updated but still valid review on the effect of IVIg in MMN

76. Azulay J-P, Blin O, Pouget J, et al. Intravenous immunoglobulin treatment in patients with motor neuron syndromes associated with anti-GM1 antibodies: a double-blind, placebo controlled study. Neurology. 1994;44:429-32.

77. Van den Berg LH, Kerkhoff H, Oey PL, et al. Treatment of multifocal motor neuropathy with high dose intravenous immunoglobulins: a double blind, placebo controlled study. J Neurol Neurosurg Psychiatry. 1995;59:248-52.

78. Federico P, Zochodne DW, Hahn AF, et al. Multifocal motor neuropathy improved by IVIg. Randomized, double-blind, placebo-controlled, study. Neurology. 2000;55:1257-62.

79. Léger J-M, Chassande B, Musset L, et al. Intravenous immunoglobulin therapy in multifocal motor neuropathy: a double-blind placebo-controlled study. Brain. 2001;124:145-153.

80. Hahn AF, Beydoun SR, Lawson V, et al. A phase III randomized, placebo-controlled study of the efficacy and safety of $10 \%$ liquid intravenous immunoglobulin (IVIg) for the treatment of multifocal motor neuropathy. J Peripher Nerv Syst. 2012;17:243-4.

81. Azulay JP, Rihet P, Pouget J, et al. Long-term follow-up of multifocal motor neuropathy with conduction block under treatment. J Neurol Neurosurg Psychiatry. 1997;62:391-394.

82. Van den Berg-Vos RM, Franssen H, Wokke JHJ, et al. Multifocal motor neuropathy: longterm clinical and electrophysiological assessment of intravenous immunoglobulin maintenance treatment. Brain. 2002;125:1875-86.

83. Vucic S, Black KR, Chong PS, et al. Multifocal motor neuropathy. Decrease in conduction blocks and reinnervation with long-term IVIg. Neurology. 2004;63:1264-9. 
84. Léger JM, Viala K, Cancalon F, et al. Intravenous immunoglobulin as short- and long-term therapy of multifocal motor neuropathy: a retrospective study of response to IVIg and of its predictive criteria in 40 patients. J Neurol Neurosurg Psychiatry. 2008;79:93-96.

85. Terenghi F, Cappellari A, Bersano A, et al. How long is IVIg effective in multifocal motor neuropathy? Neurology. 2004;62:666-8.

86. Harbo T, Andersen H, Hess A, Hansen et al. Subcutaneous versus intravenous immunoglobulin in multifocal motor neuropathy: a randomized single-blinded cross-over trial. Eur J Neurol. 2009;16: 631-8.

87. Eftimov F, Vermeulen M, de Haan RJ, et al. Subcutaneous immunoglobulin therapy for multifocal motor neuropathy. J Peripher Nerv Syst. 2009;14:93-100

88. * Umapathi T, Hughes R, Nobile-Orazio E, et al. Immunosuppressant and immunomodulatory treatments for multifocal motor neuropathy. Cochrane Database Syst Rev. 2015;4:CD003217.

This is a recent and updated review on the effect of immune suppressive therapy in MMN.

89. * Piepers S, Van den Berg-Vos R, Van der Pol W-L, et al. Mycophenolate mofetil as adjunctive therapy for MMN patients: a randomized, controlled trial. Brain. 2007;130:200410.

This is the first randomized controlled study with immune suppressant in MMN showing that mycophenolate mofetil is well tolerated in this disease but does not help to reduce the dose or to enhance the effect of IVIg in MMN.

90. Nobile-Orazio E, Terenghi F, Cocito D, et al. Oral methotrexate as adjunctive therapy in patients with Multifocal Motor Neuropathy on chronic IVIg therapy. J Peripher Nerv Syst. 2009;14:203-5. 
91. Fitzpatrick AM, Mann CA, Barry S, et al. An open label clinical trial of complement inhibition in multifocal motor neuropathy. J Peripher Nerv Syst. 2011;16:84-91

92. Stielgbauer K, Topakian R, Hinterberger G, et al. Beneficial effect of rituximab therapy in multifocal motor neuropathy. Neuromusc Disord. 2009;19:473-5.

93. Chaudhry V, Cornblath DR. An open label trial of rituximab (Rituxan ${ }^{\circledR}$ ) in multifocal motor neuropathy. J Peripher Nerv Syst. 2010;15:196-201.

94. Nobile-Orazio E. Neuropathy and monoclonal gammopathy. In: Handbook of Clinical Neurology. Vol. 115 (3rd series); Peripheral Nerve Disorders. G. Said and C. Krarup, Editors. Elsevier BV. Amsterdam, The Netherlands. 2013;115:443-59.

95. * Joint Task Force of the EFNS and the PNS. European Federation of Neurological Societies/Peripheral Nerve Society Guideline on management of paraproteinemic demyelinating neuropathy: Report of a joint task force of the European Federation of Neurological Societies and the Peripheral Nerve Society - First Revision. J Peripher Nerv Syst. 2010:15:185-195.

This guideline on the diagnosis and treatment of neuropathy associated with monoclonal gammopathy provide a valid clue for the diagnosis and therapy of this neuropathy.

96. Nobile-Orazio E, Meucci N, Baldini L, et al. Long-term prognosis of neuropathy associated with anti-MAG IgM M-proteins and its relation with immune therapies. Brain. 2000;123:710-7.

97. Niermeijer JMF, Fischer K, Eurelings M, et al. Prognosis of polyneuropathy due to IgM monoclonal gammopathy. Neurology. 2010;74:406-12.

98. ** Lunn MP, Nobile-Orazio E. Immunotherapy for IgM anti-myelin-associated glycoprotein paraprotein-associated peripheral neuropathies. Cochrane Database Syst Rev. 2016;10:CD002827. 
This very recent review analyses the effect of immune therapies in anti-MAG neuropathy and shows that, by combining the results of two small negative studies, rituximab proves to be indeed effective in this neuropathy.

99. Pestronk A, Florence J, Miller T, et al. Treatment of IgM antibody associated polyneuropathies using rituximab. J Neurol Neurosurg Psychiatry. 2003;74:485-489.

100.Renaud S, Fuhr P, Gregor M, et al. High-dose rituximab and anti-MAG associated polyneuropathy. Neurology. 2006;66:742-4.

101.Benedetti L, Briani C, Grandis M, et al. Predictors of response to rituximab in patients with neuropathy and anti-myelin associated glycoprotein immunoglobulin M. J Periph Nerv Syst. 2007;12:102-107.

102.Niermeijer JM, Eurelings M, Lokhorst HL, et al. Rituximab for polyneuropathy with IgM monoclonal gammopathy. J Neurol Nerosurg Psychiatry. 2009;80:1036-1039.

103.* Dalakas MC, Rakocevic G, Salajegheh M, et al. Placebo controlled trial of rituximab in IgM anti-myelin-associated glycoprotein antibody demyelinating neuropathy. Ann Neurol. 2009;65:286-293.

This randomized controlled study show that rituximab may have some effect in anti-MAG neuropathy even if the results only derives by the exclusion of an erroneously included patient.

104.* Léger JM, Viala K, Nicolas G, et al. Placebo controlled trial of rituximab in IgM antimyelin-associated glycoprotein neuropathy. Neurology. 2013;80(24):2217-25.

This negative controlled study on the effect of rituximab in anti-MAG neuropathy shows that the therapy may improve some parameters and, combined with the previous study, permits to show that the therapy is indeed effective in this neuropathy. 
105.Benedetti L, Briani C, Franciotta D, et al. Long-term effect of rituximab in anti-MAG polyneuropathy. Neurology. 2008;71:1742-4.

106. Carson KR, Evens AM, Richey EA, et al. Progressive multifocal leukoencephalopathy following rituximab therapy in HIV negative patients: a report of 57 cases from the Research on Adverse Drug Event and Reports project. Blood. 2009;113:4834-4840.

107.Gruson B, Ghomari K, Beaumont M, et al. Long-term response to rituximab and fludarabine combination in IgM anti-myelin-associated glycoprotein neuropathy. J Peripher Nerv Syst. 2011;16:180-5.

108.Gomez A, Hoffman JE. Anti myelin-associated-glycoprotein antibody peripheral neuropathy response to combination chemoimmunotherapy with bendamustine/rituximab in a patient with biclonal IgM $\mathrm{k}$ and $\operatorname{IgM} \lambda$ : case report and review of the literature. Clinical Lymphoma, Myeloma \& Leukemia. 2016;16(7): e101-8.

109. Codron P, Cousin M, Subra JF, et al. Therapeutic plasma exchange in chronic dysimmune peripheral neuropathies: A 10-year retrospective study. J Clin Apher. 2017 Mar 1; doi: 10.1002/jca.21530. [Epub ahead of print]

110.Nobile-Orazio E, Gallia F, Terenghi F, et al. How useful are anti-neural IgM antibodies in the diagnosis of chronic immune-mediated neuropathies? J Neurol Sci. 2008; 266:156-63.

111. Magy L, Kaboré R, Mathis S, et al. Heterogeneity of Polyneuropathy Associated with AntiMAG Antibodies. J Immunol Res. 2015, Article ID 450391.

112.Gallia F, Balducci C, Nobile-Orazio E. Efficacy and tolerability of different brands of intravenous immunoglobulin in the maintenance treatment of chronic immune mediated neuropathies. J Peripher Nerv Syst. 2016 ;21(2): 82-4. 